\title{
Pseudomonas aeruginosa vesicles associate with and are internalized by human lung epithelial cells Susanne J Bauman and Meta J Kuehn*
}

\author{
Address: Duke University Medical Center, Department of Biochemistry, Duke University Medical Center, Durham, NC 27710, USA \\ Email: Susanne J Bauman - sjb7@duke.edu; Meta J Kuehn* - meta.kuehn@duke.edu \\ * Corresponding author
}

Published: 3 February 2009

BMC Microbiology 2009, 9:26 doi:10.1 |86//47|-2 |80-9-26
Received: 8 July 2008

Accepted: 3 February 2009

This article is available from: http://www.biomedcentral.com/I47|-2/80/9/26

(c) 2009 Bauman and Kuehn; licensee BioMed Central Ltd.

This is an Open Access article distributed under the terms of the Creative Commons Attribution License (http://creativecommons.org/licenses/by/2.0), which permits unrestricted use, distribution, and reproduction in any medium, provided the original work is properly cited.

\begin{abstract}
Background: Pseudomonas aeruginosa is the major pathogen associated with chronic and ultimately fatal lung infections in patients with cystic fibrosis (CF). To investigate how $P$. aeruginosaderived vesicles may contribute to lung disease, we explored their ability to associate with human lung cells.
\end{abstract}

Results: Purified vesicles associated with lung cells and were internalized in a time- and dosedependent manner. Vesicles from a CF isolate exhibited a 3- to 4-fold greater association with lung cells than vesicles from the lab strain PAOI. Vesicle internalization was temperature-dependent and was inhibited by hypertonic sucrose and cyclodextrins. Surface-bound vesicles rarely colocalized with clathrin. Internalized vesicles colocalized with the endoplasmic reticulum (ER) marker, TRAP $\alpha$, as well as with ER-localized pools of cholera toxin and transferrin. CF isolates of P. aeruginosa abundantly secrete PaAP (PA2939), an aminopeptidase that associates with the surface of vesicles. Vesicles from a PaAP knockout strain exhibited a $40 \%$ decrease in cell association. Likewise, vesicles from PAOI overexpressing PaAP displayed a significant increase in cell association.

Conclusion: These data reveal that PaAP promotes the association of vesicles with lung cells. Taken together, these results suggest that $P$. aeruginosa vesicles can interact with and be internalized by lung epithelial cells and contribute to the inflammatory response during infection.

\section{Background}

Pseudomonas aeruginosa is the major pathogen associated with chronic and ultimately fatal lung infections in patients with cystic fibrosis (CF). Current research suggests that $P$. aeruginosa live anaerobically in the mucus layer of the CF lung and are rarely found in contact with epithelial cells $[1,2]$. Extracellular proteases are secreted by $P$. aeruginosa, including Las $\mathrm{A}$, elastase, alkaline protease, and protease IV, and these are known contributors to virulence in lung infections [3-5]. Like other gram negative bacteria, $P$. aeruginosa also release spheres of outer membrane known as outer membrane vesicles [6]. They consist of entrapped periplasmic components and outer membrane constituents, including lipopolysaccharide (LPS), glycerophospholipids, and outer membrane proteins (OMPs) [7]. Due to their small size, vesicles potentially gain access to host cells more easily than whole bacteria. Considering that vesicles are armed with bacterial proteases, toxins, surface adhesins and/or invasins, vesicles present a potentially significant contributor to lung damage caused by $P$. aeruginosa. Since they contain many immunostimulatory compounds, it is not surprising that $P$. aeruginosa vesicles induce a significant IL-8 response from cultured human lung cells [8]. 
Vesicles allow bacteria to disperse a complex of soluble and insoluble bacterial products into the surrounding milieu. Vesiculation appears to be a conserved process among both pathogenic and non-pathogenic bacteria and the role of outer membrane vesicles in pathogenesis is a burgeoning area of research [9]. Many pathogenic bacterial species aside from $P$. aeruginosa produce vesicles that contain toxins or other virulence factors and, in several cases, vesicles have been proposed to be vehicles for toxin delivery to eukaryotic cells [10-16]. In order to deliver toxic content, vesicles must first bind to host cells. Vesicles from Shigella flexneri [17], Borellia burgdorferi [18], Actinobacillus actinomycetemcomitans $[13,19]$ and ETEC $[14,20]$ have been observed to bind cultured host cells. Vesicles have also been observed interacting with host cells in vivo. In biopsies of infected patients, vesicles from $H$. pylori were found to bind intestinal cells $[10,21]$.

$P$. aeruginosa vesicles have been amongst the most thoroughly studied vesicles to date. They have been shown to contain the virulence factors pro-elastase, hemolysin, phospholipase $\mathrm{C}$, and alkaline phosphatase, as well as the penicillin-degrading enzyme $\beta$-lactamase and the Pseudomonas quorum sensing signal (PQS) and other hydroxyalkylquinolones [22-24]. We recently reported that the secreted aminopeptidase, PaAP, is enriched in outer membrane vesicles that we purified from each of the tested CF strains of $P$. aeruginosa [8]. Interestingly, PaAP expression was found to increase 21-fold when PAO1 was grown under microaerobic conditions [25], and 103-fold when it was grown in the presence of primary lung epithelial cells [26]. An analogous zinc metalloprotease was discovered to be associated with vesicles produced by a different CF pathogen, Burkholderia cepacia, and a strain with a knockout in this gene was less virulent in an animal model [27]. Such studies have stimulated much interest in determining how vesicles and vesicle components contribute to $P$. aeruginosa infection and disease in the lungs.

In this study, we use both cultured and primary airway epithelial cells to investigate vesicle-host cell interactions and to assess the contribution of PaAP to this interaction. We report that $P$. aeruginosa vesicles are internalized by human lung cells and PaAP increases vesicle association with lung cells. The results point to physiological roles for $P$. aeruginosa $\mathrm{PaAP}$ and vesicles during an infection.

\section{Results}

P. aeruginosa vesicle association with lung epithelial cells is strain-dependent

We examined whether vesicles from various $P$. aeruginosa isolates would associate with cultured human respiratory epithelial cells. Fluorescently labeled vesicles (FITC-vesicles) from late log-phase cultures were incubated with A549 human lung epithelial cells and the amount of ves- icles associated with host cells after incubation at $37^{\circ} \mathrm{C}$ was quantitated using a previously established microtiter plate assay [14]. To account for minor variability in the fluorescent labeling of vesicles, the amount of cell-associated vesicles was extrapolated from standard curves relating fluorescence to ng of FITC-vesicles for each of the vesicle preparations. Cell-associated fluorescence increased over time for vesicles for each of the P. aeruginosa isolates, however significantly more (3.3-fold) vesicles from the CF isolate S470 associated with A549 cells compared with PAO1 vesicles (Fig. 1A). The cell association profile for vesicles from another CF isolate, CF2, was very similar to the one exhibited by $\mathrm{S} 470$, and host cell association of vesicles from all isolates was dose-dependent (data not shown).

To test whether the vesicles would interact similarly with primary cells, we incubated vesicles with human bronchial epithelial (HBE) cells from healthy human volunteers (Fig. 1B). The results for the HBE cells were similar to those with cultured cells, thus cultured cells appeared to be a good model for primary cells in further assays. Together, these data indicate that $P$. aeruginosa vesicles from CF strains associate to a greater extent with epithelial cells than vesicles from a non-CF strain.

When we tested temperature dependence of vesicle-host cell association we found that incubation at $4{ }^{\circ} \mathrm{C}$ substantially decreased the amount of $S 470$ vesicles associated with the lung cells, whereas little-to-no difference was observed for PAO1 vesicles (Fig. 2A). These data indicate that a temperature-dependent mechanism was responsible for the differences observed in the association between vesicles from a CF strain and vesicles from a non-CF strain.

\section{Pseudomonas aeruginosa vesicles are trafficked into lung epithelial cells}

Temperature-dependent association of $\mathrm{S} 470$ vesicles suggested that these vesicles may be internalized by the lung epithelial cells. We used confocal microscopy to analyze vesicle-host cell interactions. Cultured A549 cells were incubated with FITC-labeled $\$ 470$ vesicles for 6 hours at $37^{\circ} \mathrm{C}$, and plasma membranes were stained with AF633wheat germ agglutinin (WGA) to visualize cell boundaries. At $37^{\circ} \mathrm{C}$, vesicle fluorescence appeared to be mostly internal and concentrated in a perinuclear region of the cell (Fig. 2B). Very little vesicle association was observed for incubations maintained at $4{ }^{\circ} \mathrm{C}$ (Fig. 2C). Thus, both binding and internal localization of $\mathrm{S} 470$ vesicles was affected at the lower temperature.

To further confirm vesicle internalization, vesicles were labeled using AF488 instead of FITC to maximize fluorescence and minimize the effects of photobleaching. Many 
A
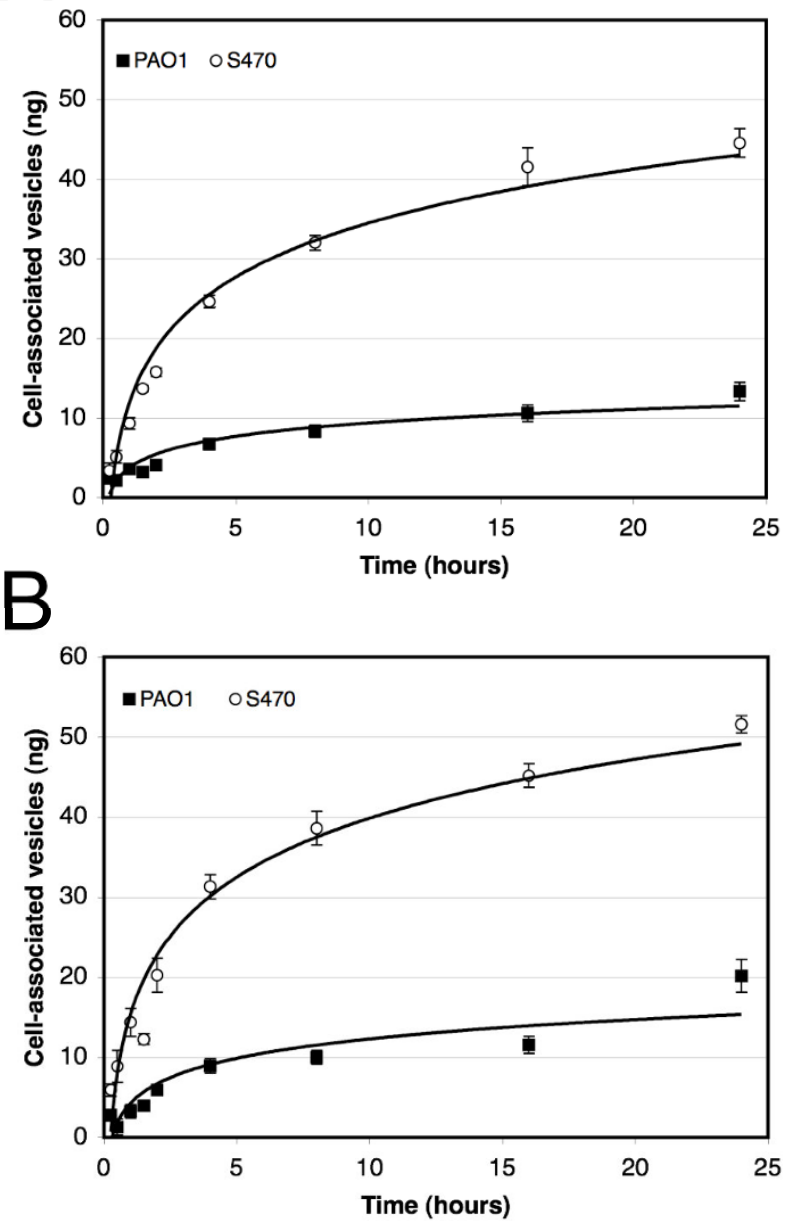

Figure I

Vesicles from a CF isolate associates to a greater extent with lung cells compared to PAOI vesicles. FITC-labeled vesicles ( $2.5 \mu \mathrm{g}$ per well) were incubated with confluent monolayers of approximately $5 \times 10^{4}$ A549 cells $(A)$ or HBE cells $(B)$ for indicated times at $37^{\circ} \mathrm{C}$. Fluorescence associated with washed, solubilized cells was quantitated and correlated to vesicle amount using standard curves generated for vesicles from each strain. Experiments were done in triplicate, SEM is indicated for 2 to 7 separate experiments. At the $24 \mathrm{~h}$ time point, $\mathrm{p}<0.00 \mathrm{I}$ for each data set.

vesicle components are labeled by this reagent (Fig 3A), indicating that the fluorescent signal would not track merely a single component. After incubating AF488-S470 vesicles with A549 cells for $1 \mathrm{~h}$ at $37^{\circ} \mathrm{C}$, the surface of cell monolayers was labeled with a membrane-impermeable biotin. The biotinylated surface was then detected using AF633-streptavidin and cell fluorescence was visualized by confocal microscopy. As a result, surface-exposed vesicles appear white and internalized vesicles appear green in an overlay of streptavidin and vesicle fluorescence. After a 1 hour incubation with A549 cells, mainly green, perinu- clear fluorescence was observed (Fig 3B), with only a few white, surface localized vesicles (indicated by arrows, Fig $3 \mathrm{~B})$, indicating that $\mathrm{S} 470$ vesicles are internalized by lung cells.

To investigate the mode of $P$. aeruginosa vesicle internalization, we treated cells with common inhibitors of endocytic pathways. Filipin, chlorpromazine, cytochalasin D, and $\mathrm{NiCl}_{2}$ did not inhibit uptake (data not shown). Pretreatment of cells with methyl- $\beta$-cyclodextrin $(\mathrm{M} \beta \mathrm{CD})$, which removes cholesterol from membranes, inhibited vesicle uptake, however, preincubation with methyl- $\alpha$ cyclodextrin, which typically is used as a negative control for $M \beta C D$, inhibited vesicle uptake as well (Fig. 3C and 3D). Inhibition of vesicle uptake was also achieved using hypertonic sucrose (Fig 3E). In parallel control incubations, we pretreated vesicles with hypertonic sucrose or cyclodextrins instead of pretreating the lung cells. In these controls, vesicles were still readily internalized (data not shown), indicating that the inhibition of vesicle uptake was due to effects on the lung cells and not on the vesicles themselves.

Since we observed the greatest effect on vesicle internalization using hypertonic sucrose and $M \beta C D$, which impair clathrin-coated pit formation and invagination, respectively $[28,29]$, we next investigated whether vesicles would colocalize with clathrin. We observed a few locations where vesicle fluorescence colocalized with clathrin on the cell surface, however vesicle fluorescence did not co-localize with any intracellular clathrin (Fig 4). Vesicles did not colocalize with any caveolin, however it should be noted that very little caveolin was visualized in the A549 cells, consistent with reports of low levels of caveolin-1 expression in these cells [30,31] (data not shown). These data suggest that vesicles may be associated with clathrincoated pits, but only transiently, at an early stage in the active uptake process.

\section{Internalized vesicle components colocalize with the endoplasmic reticulum}

We repeatedly observed internalized vesicle-associated fluorescence localized to a perinuclear region. We examined whether vesicles were trafficked to the same compartments as transferrin and cholera toxoid (CTB). Only transferrin and CTB that were perinuclear colocalized with internalized vesicles, whereas the majority of cytosolic compartments containing transferrin and CTB did not [see Additional file 1]. To determine whether this perinuclear region corresponded to the endoplasmic reticulum (ER), we treated cells with the glycoside digitonin, which, at low concentrations, permeabilizes the plasma membrane and releases cytosolic proteins but preserves the ER membrane [32,33]. After digitonin treatment, cells that had lost the cytoplasmic marker, $\beta$-tubulin, still retained a perinuclear halo of vesicle-associated fluorescence (data 

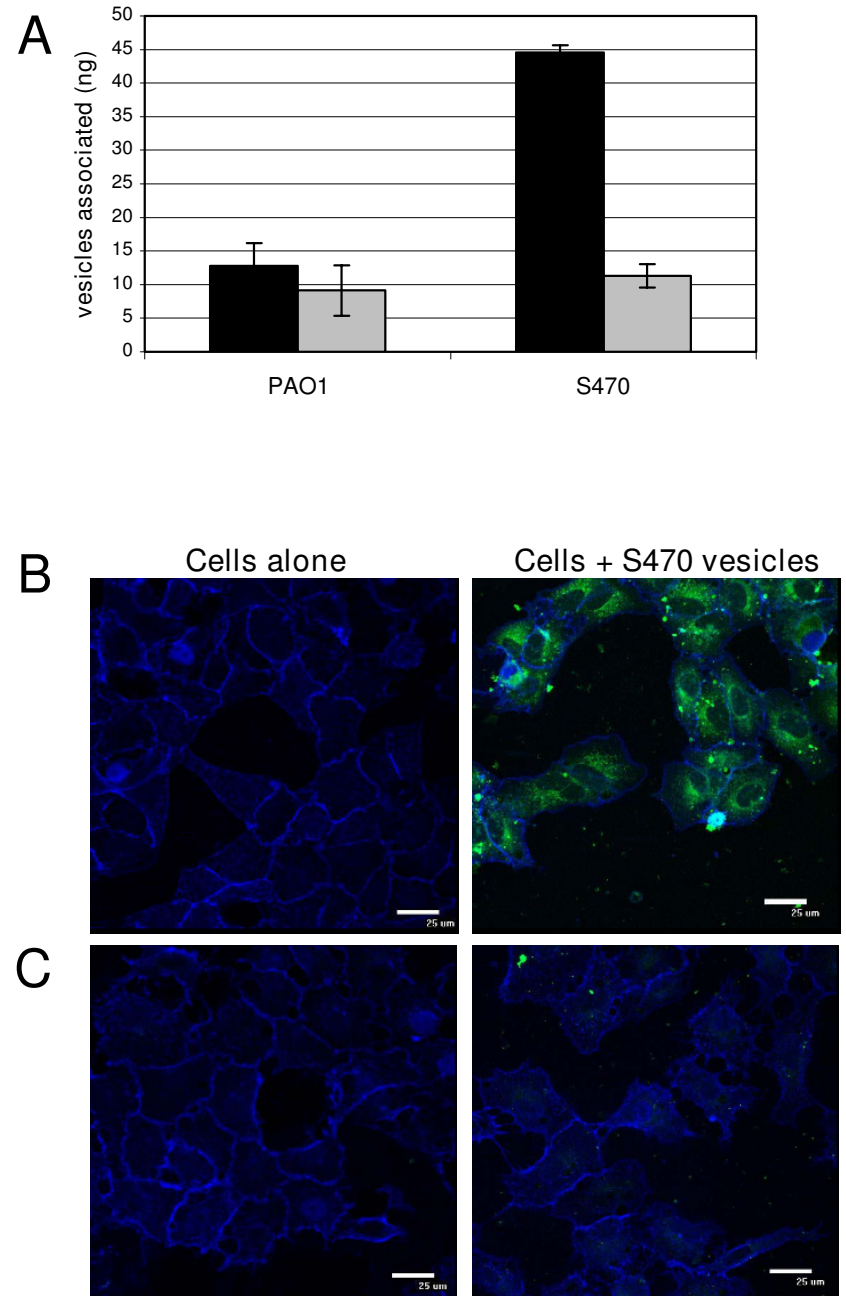

Figure 2

$\mathbf{S 4 7 0}$ vesicle association with host cells is temperature-dependent. A, FITC-labeled-vesicles ( $2.5 \mu \mathrm{g}$ per well) were incubated with A549 cells $\left(5 \times 10^{4}\right.$ cells per well) for 24 $\mathrm{h}$ at $37^{\circ} \mathrm{C}$ (black bars), or $4^{\circ} \mathrm{C}$ (gray bars). SEM is indicated, $\mathrm{n} \geq 2$, in triplicate. B and C, A549 cells alone (left panels) or incubated with $2.5 \mu \mathrm{g}$ FITC-labeled S470 vesicles (green, right panels) for $6 \mathrm{~h}$ at $37^{\circ} \mathrm{C}(\mathrm{B})$ or $4^{\circ} \mathrm{C}(\mathrm{C})$. After incubation, cells were washed, labeled with AF633-WGA (blue), fixed in $2 \%$ paraformaldehyde, and visualized by confocal microscopy.

not shown). In these treated cells, vesicle fluorescence clearly colocalized with the integral ER membrane protein TRAP $\alpha$ (Fig. 5). These data suggest that internalized vesicle components traffic to the ER within 1 hour of exposure.

\section{PaAP promotes vesicle association with human respiratory epithelial cells}

We wondered whether host cell association depended on $\mathrm{PaAP}$, one of the major protein components of vesicles derived from CF isolates (Fig 6A). Quantitative 2D-DIGE revealed PaAP is at least 65 -fold enriched in $\mathrm{S} 470$ vesicles compared with PAO1 vesicles [8]. To test whether PaAP on vesicles contributed to host cell association, we overexpressed PaAP in PAO1 (PAO1/pS41) and constructed a deletion in the aminopeptidase in strain $\mathrm{S} 470$ (S470APKO5). We confirmed that purified PAO1/pS41 vesicles were enriched in PaAP compared with PA01 vesicles and that S470APKO5 did not contain detectable amounts of PaAP [see Additional file 2]. Purified PAO1/ pS41 vesicles associated with A549 cells more than twice as much as PA01 vesicles, whereas $\mathrm{S} 470 \mathrm{APKO} 5$ vesicles associated $40 \%$ less with the lung cells than $\mathrm{S} 470$ vesicles (Fig. 6B, C). Unfortunately, complementation of S470APKO5 was not successful since vesicles from S470APKO5 expressing PaAP from pS41 contained approximately 10-fold less PaAP and had 10-fold less aminopeptidase activity than S470 vesicles [see Additional file 3, parts A and B]. Induction of PaAP expression in S470APKO5 did not help correct the complementation defect and increase the level of vesicle-bound PaAP, although the total amount of PaAP in the supernatant was equivalent to that of S470 [see Additional file 3, part C]. As a result, it was not surprising that S470APKO5/pS41 vesicles associated with host cells to approximately the same extent as those from APKO5 (data not shown). Collectively, these data support a dose-dependent contribution of PaAP to the association of vesicles with host cells.

\section{Discussion}

With these results, we have revealed several facets of interactions between $P$. aeruginosa vesicles and human lung epithelial cells. We have demonstrated that $P$. aeruginosa vesicles are internalized by epithelial cells and trafficked intracellularly so that vesicle components accumulate in the ER. We have also shown that PaAP, an enzyme more abundant in vesicles produced by many CF isolates compared with non-clinical isolates, significantly contributes to the interaction of $P$. aeruginosa vesicles with host cells.

Internalization by host cells has been reported to occur for outer membrane vesicles from numerous species. For instance, our lab has shown previously that ETEC vesicles are internalized in an LT-dependent fashion via ganglioside $\mathrm{G}_{\mathrm{M} 1}$ in caveolin-enriched lipid rafts of epithelial cells [20]. Additionally, fluorescent beads coated with Porphyromonas gingivalis vesicles also colocalized with caveolin and $\mathrm{G}_{\mathrm{M} 1}$ and were internalized by HeLa cells in a cholesterol and dynamin-dependent manner [34]. Our observations regarding the temperature-dependent extent and location of vesicle-associated fluorescence in host cells and decreased fluorescence in host cells upon pretreatment with methyl- $\beta$-cyclodextrin (which disrupts caveolae, lipid rafts, as well as clathrin-coated pit-mediated entry pathways) suggested that $\mathrm{S} 470$ vesicles were also internalized. 


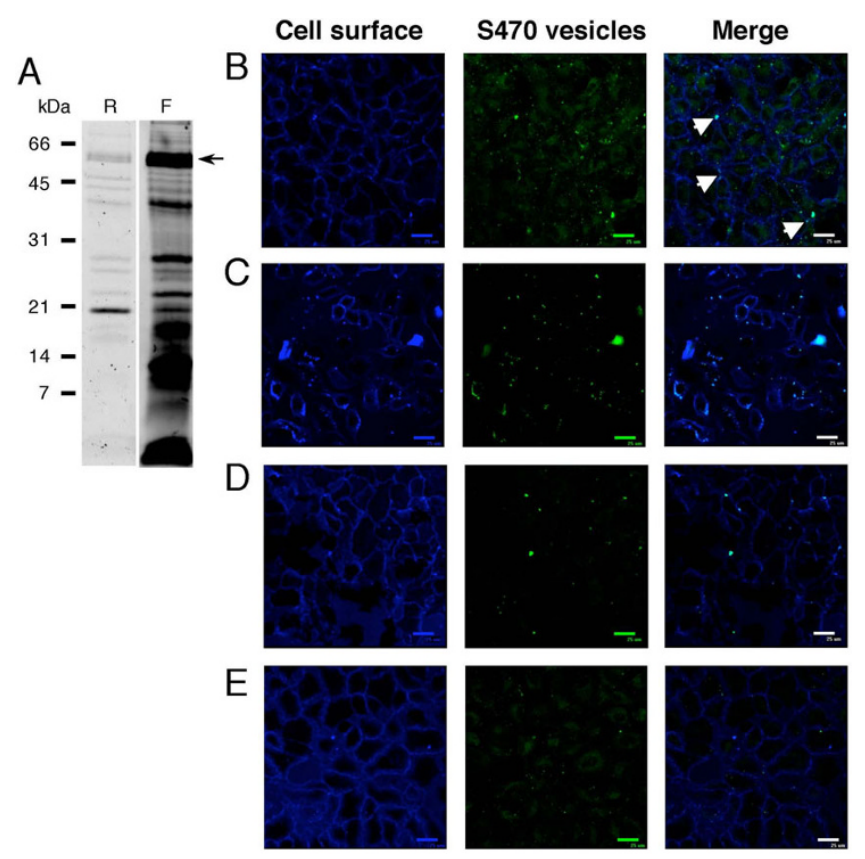

Figure 3

Vesicle components are internalized by lung cells, and internalization is inhibited by hypertonic sucrose and cyclodextrins. A, SDS-PAGE gel profiles of S470 vesicles before and after AF488 labeling. Total protein in unlabeled vesicles was visualized after SYPRO Ruby staining of the gel (R). AF488-labeled proteins were visualized by placing the unstained gel on a UV lightbox $(F)$. The migration of molecular weight standards ( $\mathrm{kDa}$ ) and $\mathrm{PaAP}$ (arrow) is indicated. B, A549 cells incubated with $2.5 \mu \mathrm{g}$ AF488-labeled $\mathrm{S} 470$ vesicles (green) for I h at $37^{\circ} \mathrm{C}$. Cell surface was labeled using biotin and AF633-streptavidin (blue), fixed in $2 \%$ paraformaldehyde, and visualized by confocal microscopy. A549 cells were pretreated with $10 \mathrm{mM}$ methyl- $\beta$-cyclodextrin (C), $10 \mathrm{mM} \alpha$-cyclodextrin (D), or $0.45 \mathrm{M}$ sucrose (E), for 30 minutes, and then incubated with $2.5 \mu \mathrm{g}$ AF488labeled $\mathrm{S} 470$ vesicles (green) for I h at $37^{\circ} \mathrm{C}$. Cell surface was labeled using biotin and AF633-streptavidin (blue), fixed in $2 \%$ paraformaldehyde, and visualized by confocal microscopy. Bars indicate $25 \mu \mathrm{m}$.

In contrast to other examples of internalized vesicles, $P$ aeruginosa vesicles appear to enter host cells via multiple pathways. Hypertonic media, which impairs clathrin coated pit formation, did significantly decrease vesicle internalization and some surface-bound vesicles were found colocalized with clathrin. However, neither treatment with filipin, which disrupts lipid rafts, nor chlorpromazine, which blocks clathrin-coated pits, decreased vesicle internalization significantly. It should also be considered that $P$. aeruginosa vesicles could fuse with the epithelial cells and that vesicle membrane components are subsequently internalized by plasma membrane trafficking while lumenal components are liberated into the host cell cytosol. Evidence of fusion of vesicles with the plasma membrane has been presented for Actinobacillus actinomycetemcomitans vesicles [13]: Confocal microscopy of HL60 cells coincubated with these vesicles showed immediate and strong labelling, primarily at the plasma membrane. We did not observe strong perimeter labelling of host cells with $P$. aeruginosa vesicles (Fig $2 \mathrm{~B}$ ). In fact, when we blocked active transport with hypertonic sucrose, we found a significant decrease in vesicle-associated fluorescence, not accumulation of fluorescence at the cell periphery (Fig 3E). Thus, our data support a model where $P$. aeruginosa vesicles do not fuse to the plasma membrane, but instead bind and are internalized.

We observed an increase in human lung epithelial cellassociated fluorescence over time. This result is consistent with either vesicle attachment causing receptor upregulation, or continuous vesicle binding, internalization, recycling of vesicle receptors to the cell surface. These characteristics are similar to the behavior of enterotoxigenic E. coli vesicles with intestinal epithelial cells [14]. Further experiments using different inhibitors, markers, and cell lines, will be necessary to definitively identify the host cell factors critical to $P$. aeruginosa vesicle entry. In relation to CF-related research, it would be particularly interesting to see whether the interactions depend on functional and properly localized CFTR. Ceramide-rich rafts containing clusters of the CFTR and CD95 have been implicated as the means for internalization of whole $P$. aeruginosa. These rafts are disrupted by $M \beta C D$, and thus in light of our $M \beta C D$ treatment results, they present a potential route for vesicle internalization [35].

Once internalized, vesicles may be sequestered, degraded, or their components may be dispersed, with their membranes disrupted and/or fused with the endocytic membrane. In the case of $S$. flexneri vesicles, for instance, vesicle lumenal content was found in the host cell cytosol after vesicles were phagocytosed to a non-acidified compartment by Henle 407 epithelial cells [36]. We show that $P$. aeruginosa vesicle-associated intracellular fluorescence is concentrated to bright puncta and do not encounter an acidified compartment, since vesicle-associated FITC fluorescence (which is $\mathrm{pH}$ sensitive) is not quenched, even in long incubations (Fig 1). Notably, a significant amount of vesicle-associated fluorescence colocalized with the integral ER membrane protein TRAP $\alpha$, even after a relatively brief incubation time. Transferrin and CT eventually route to the ER, and indeed, those pools of Transferrin and CT that had reached the ER colocalized with the vesicle fluorescence. None of the currently identified $P$. aeruginosa vesicle proteins have an ER retention sequence to direct the trafficking of these bacterial factors to the ER (such as the case for LT which has RDEL at its C-terminus). Since intracellular trafficking of S470APKO5 vesicles was not 


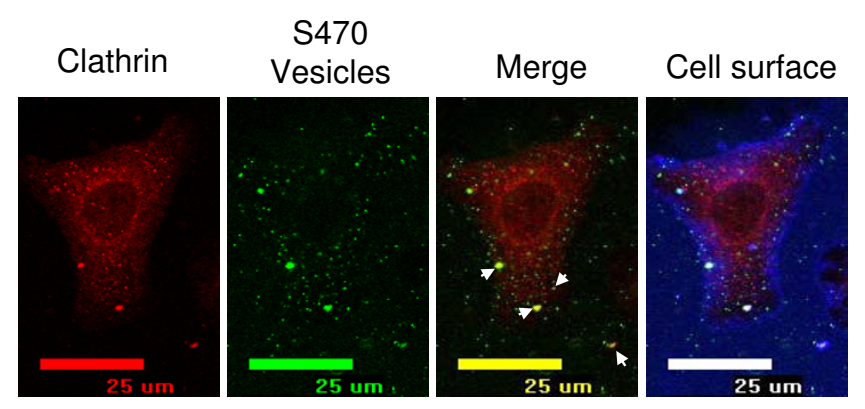

Figure 4

Vesicles rarely co-localize with surface-associated clathrin. AF488-S470 vesicles $(2.5 \mu \mathrm{g})$ were incubated with A549 cells for I h at $37^{\circ} \mathrm{C}$. Cell surface was labeled using biotin and AF633-streptavidin (blue). Cells were washed, fixed, permeabilized, and probed with mouse anti-clathrin antibodies and AF555-labeled goat anti-mouse secondary antibody. Arrows indicate very occasional colocalization of clathrin and vesicle fluorescence at the cell surface.

noticeably different from $\mathrm{S} 470$ vesicles (data not shown), internalized vesicle trafficking appears to be PaAP-independent. In all, many questions remain regarding the trafficking of $P$. aeruginosa vesicle membrane and lumenal content after endocytosis, and this area deserves further exploration.

In some cases the factor on bacterial vesicles responsible for host cell binding has been identified as a virulence factor [9]. For example, the heat-labile enterotoxin (LT) is bound to the surface of ETEC vesicles, and vesicle-bound LT mediates vesicle binding to cultured eukaryotic cells via the $\mathrm{LT}$ receptor, ganglioside $\mathrm{G}_{\mathrm{M} 1}[11,14]$. In contrast, leukotoxin transported in A. actinomycetemcomitans vesicles was not responsible for vesicle association with HL60 cells [13]. We have found that PaAP also is located on the vesicle surface (preliminary data), and that host cell association correlated with PaAP levels on the vesicles. Strains overexpressing PaAP or deleted in PaAP, respectively, produced vesicles that associated to a greater or lesser extent than vesicles from the corresponding isogenic parent strains. A direct correlation between vesicle association and PaAP levels also held for strains naturally expressing PaAP at different levels. PaAP expression is highly regulated and typically does not occur until stationary phase [37-40]. This was true for our cultures of PAO1, and as a result PaAP was nearly absent from PAO1 vesicles purified from late log-phase cultures (see Fig 6 and [Additional file 2, Part A]). In contrast, strain S470 begins to express PaAP in late log phase, therefore PaAP was enriched in the late log-phase S470 vesicles (see Fig 6 and [Additional file 2, Part A]). Correspondingly, PAO1 vesicles associated 3-4 fold less than $\mathrm{S} 470$ vesicles (Fig 1). Thus, the degree of bacterial vesicle-host cell interaction can depend on the growth phase of the parent bacterium. Despite the importance of PaAP, it is not the only factor governing host cell association since association by $\mathrm{S} 470 \mathrm{APKO} 5$ vesicles was only reduced by $40 \%$ compared with $\mathrm{S} 470$ vesicles. The conclusion that $P$. aeruginosa vesicles can utilize numerous internalization pathways is consistent with our finding that factors other than PaAP are involved in vesiclehost cell association.

We describe that PaAP expression in trans failed to complement the PaAP deletion with regards to the ability to obtain WT levels of vesicle-localized PaAP, and hence its ability to restore WT levels of vesicle association with host cells. Complemented PaAP was expressed and secreted into the culture supernatant at WT levels, however it was not found in the vesicle-associated fraction [see Additional file 3]. In fact, overexpression of PaAP in the null mutant resulted in reduced viability (unpublished data). This lack of functional complementation is not unprecedented. Two other secreted $P$. aeruginosa proteases (LasA and protease IV) have knockout phenotypes which could not be complemented by expression of the gene from a plasmid or even from a chromosomal insertion [41-43]. The lack of complementation by the plasmid-expressed PaAP in the APKO5 PaAP knockout strain demonstrates the likelihood of a fine-tuned regulatory process that is critical for optimal PaAP expression, processing, stability, and/or secretion. Indeed, PaAP has been found to undergo complex post-translational processing ((D. FitzGerald, personal communication, and [44]).

Since vesicle-associated PaAP has activity as a zincdependent protease, PaAP could act as a proteolytic factor that exposes vesicle receptors on the host cell surface. In an attempt to test this, we constructed a mutant PaAP which lacked active site residues however it was not secreted (preliminary data). Interestingly, this suggests PaAP must bind zinc for it to fold correctly and folding is critical for export of Type 2 secretory pathway substrates. As a result, we have not yet been able to test whether PaAP activity is important in mediating host cell interactions, internalization, or trafficking.

We discovered several characteristics of PaAP expression relevant to the virulence of $P$. aeruginosa in the CF lung. First, strains taken from patients with CF express PaAP abundantly. Second, we found that more PaAP is detectable in vesicles produced by PA01 that contain the $\beta$-lactamase-resistant vector pMMB66EH than in those produced by PA01 [see Additional file 2, part A]. The association of these vesicles with lung cells was consistent with the previously described trend: PAO1/pMMB66EH vesicles associated with host cells to a greater extent compared to PA01 vesicles [see Additional file 2, part B]. The increase in PaAP expression in this case appears to be due to the pres- 

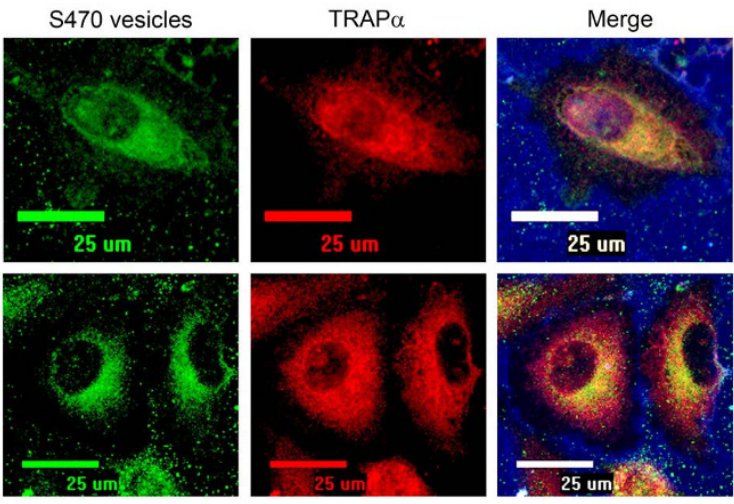

\begin{abstract}
Figure 5
Vesicles co-localize with the endoplasmic reticulum marker TRAP $\alpha$. AF488-S470 vesicles $(2.5 \mu \mathrm{g})$ were incubated with A549 cells for I hour at $37^{\circ} \mathrm{C}$. Cell surface was labeled using biotin and AF633-streptavidin (blue). Cells were washed, fixed, permeabilized with $0.015 \%$ digitonin to release cytoplasm, and probed with anti-TRAP $\alpha$ primary antibody and AF555-labeled secondary antibody.
\end{abstract}

ence of antibiotics in the media, which is particularly relevant considering that bacteria in the CF lungs are frequently exposed to antibiotics. Finally, sera from CF patients contained antibodies to several vesicle proteins, and a subset of patients ( 3 out of 13) produced antibodies to PaAP indicating that PaAP is expressed and secreted in CF patients (Fig. 7). These findings suggest that the conditions present in infected CF lungs promote upregulation of $P$. aeruginosa PaAP and production of vesicles that contain PaAP.

\section{Conclusion}

Purified $P$. aeruginosa vesicles associate with human lung cells and are internalized in a time- and dose-dependent manner. Vesicles from a CF isolate exhibit greater association with lung cells than vesicles from a lab strain. Vesicle internalization is temperature-dependent and inhibited by hypertonic sucrose and cyclodextrins. Vesicles also appear to be very transiently associated with clathrincoated pits as part of an active uptake process. After internalization, vesicle components were found to colocalize with the ER. Tested CF isolates of $P$. aeruginosa abundantly secrete PaAP, an aminopeptidase which is a major contributor to lung cell association. Therefore, our results suggest that $P$. aeruginosa vesicles can interact with and be internalized by lung epithelial cells and thereby contribute to the inflammatory response during infection.

\section{Methods}

\section{Bacterial strains and reagents}

$P$. aeruginosa strains used were the laboratory strain PA01 (Pf1 phage-cured from our lab collection), the soil isolate
ATCC 14886 (American Type Culture Collection, isolated prior to 1958), and minimally passaged, non-mucoid cystic fibrosis clinical isolates CF2, CF3, CF4, and S470 (Duke University Hospital). A549 human lung epithelia carcinoma cells were grown according to ATCC specifications in Kaighn's F-12K media containing $10 \%$ fetal bovine serum plus penicillin/streptomycin/fungizone. Human bronchial epithelial (HBE) cells were derived from anonymous healthy human volunteers. HBE cells were maintained in Bronchial Epithelial Cell Growth Media supplemented with thyroid extract. Unless indicated, all reagents were purchased from VWR.

\section{Construction of PAOI overexpressing PaAP (PAO I/pS4 I)}

The PA2939 gene encoding PaAP was amplified from strain S470 using the primers given in Table 1, which added an EcoRI site to the 5 ' end of the sequence a HindIII site to the 3' end of the sequence. The gene was subcloned into pBluescript and then moved to pMMB66EH (provided by Erich Lanka) to make plasmid pS41. Plasmid pS41 was moved into PA01 by triparental mating as described [45], using HB101/pS41 as the donor strain and MK616 (containing pRK2013) as the helper strain. Successful conjugants were selected on Pseudomonas Isolation Agar (PIA) supplemented with carbenicillin $(200 \mu \mathrm{g} /$ $\mathrm{ml}$ ) and verified by restriction digestion of miniprepped DNA.

\section{Construction of PA2939 knockout in S470 (strain APKO5)}

The PA2939 knockout vector (pAPKO) was constructed by interrupting the PA2939 sequence with a Tet cassette. DNA sequence starting from approximately 500 bp upstream of the PA2939 start codon to 30 bp into PA2939 was amplified by PCR using the "up" primers given in Table 1, which added a SpeI site to the 5' end of the DNA and mutated the 3' end to contain an EcoRI site. The remainder of the PA2939 sequence was amplified with the "down" primers given in Table 1, which mutated the 5 ' end to contain an EcoRI site and added an XhoI site to $3^{\prime}$ end. The Tet cassette was amplified from plasmid pACYC184 using primers given in Table 1 that added EcoRI sites to both ends. The three pieces were combined sequentially using the pDrive subcloning vector (Qiagen). The final construct was cut out of pDrive using SpeI and Xhol sites and inserted into the MCS of pJQ200SK $\left(\mathrm{Gm}^{\mathrm{R}}\right.$, $\mathrm{SacB}$ ) to make plasmid pAPKO.

Triparental mating was used to introduce pAPKO into strain S470 using HB101/pAPKO as the donor strain, and MT616 as the helper strain. Successful conjugants were first selected on $1 / 2$ PIA Tet $(200 \mu \mathrm{g} / \mathrm{ml})$ and $\mathrm{Gm}(20 \mu \mathrm{g} /$ $\mathrm{ml}$ ). Bacterial colonies that had undergone homologous recombination with the DNA containing the interruption of PA2939 were then counter-selected for resistance to Tet and sensitivity 5\% sucrose and Gm. Knockout S470APKO5 was verified by PCR amplification of the 

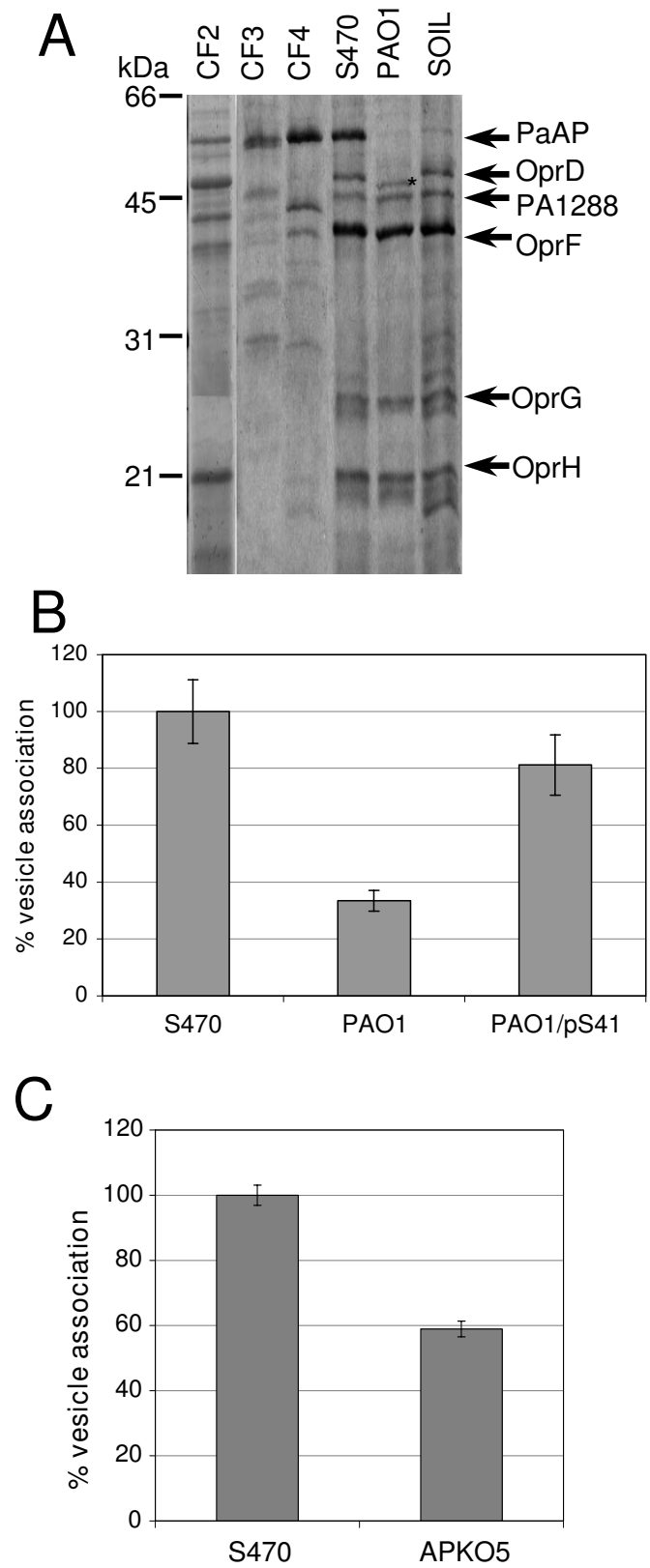

\section{Figure 6}

PaAP is abundant and active in vesicles from CF strains and promotes the association of $P$. aeruginosa vesicles with lung cells. A, Purified vesicles (approximately $10 \mu \mathrm{g})$ were TCA-precipitated and analyzed using SDS-PAGE and Coomassie staining. Previously identified proteins in PAOI vesicles and CF2 vesicles are indicated, and $(*)$ highlights the lower molecular weight form of OprD found in PAOI [8]. The migration of molecular weight standards is indicated $(\mathrm{kDa}) . \mathrm{B}$ and $\mathrm{C}$, Purified vesicles from the indicated strains $\left(2.5 \mu \mathrm{g}\right.$ protein/well) were incubated $\left(24 \mathrm{~h}, 37^{\circ} \mathrm{C}\right)$ with confluent monolayers of A549 cells $\left(5 \times 10^{4} /\right.$ well $)$ and vesicle-host cell association was compared with $\$ 470$ vesicle association within each experimental set. SEM is indicated; $n$ $=2$ in triplicate. 
were checked for sterility and filtered through $0.45 \mu \mathrm{m}$ PVDF spin filters when necessary.

\section{Vesicle association assays}

FITC-labeled vesicles $(2.5 \mu \mathrm{g}$ per well $)$ were incubated with confluent monolayers (approx. $5 \times 10^{4}$ cells per well) of A549 human lung epithelia or HBE cells in serum-free media in 96-well plates (Costar) for $15 \mathrm{~min}$ to $24 \mathrm{~h}$ at $37^{\circ} \mathrm{C}$ or $4^{\circ} \mathrm{C}$. All incubation conditions were done in triplicate. Cells were washed twice with PBS and then solubilized in $100 \mu 11 \%$ Triton X-100 in PBS. Fluorescence was quantitated using a FLUOstar Galaxy or FLUOstar Optima fluorometer (BMG Labtechnologies). A standard curve to correlate fluorescence measured in test wells to ng of vesicles was generated by adding purified FITC-labeled vesicles $(0.5 \mathrm{ng}-250 \mathrm{ng})$ from each strain to cells and immediately solubilizing the cells. Statistics were calculated using single-factor ANOVA.

\section{Confocal microscopy}

All fluorescence microscopy reagents were purchased from Molecular Probes/Invitrogen unless otherwise stated. A549 human lung epithelia $\left(1 \times 10^{5}\right.$ cells per well $)$ were seeded in LabTekII 8-well glass chamber slides (VWR) and incubated with fluorescently labeled vesicles (2.5 $\mu \mathrm{g}$ per well) in serum-free media for $1-6 \mathrm{~h}$ at $37^{\circ} \mathrm{C}$ or $4^{\circ} \mathrm{C}$. When indicated, AlexaFluor-555 transferrin $(25 \mu \mathrm{g} /$ $\mathrm{ml})$ or AlexaFluor-555 cholera toxin B subunit $(10 \mu \mathrm{g} / \mathrm{ml})$ were added to cells five minutes prior to the addition of vesicles. For inhibition experiments, cells were pretreated with inhibitors (methyl- $\beta$-cyclodextrin, $10 \mathrm{mM}$; methyl$\alpha$-cyclodextrin, $10 \mathrm{mM}$; sucrose, $0.45 \mathrm{M}$; chlorpromazine, $1 \mu \mathrm{g} / \mathrm{ml}$; filipin, $5 \mu \mathrm{g} / \mathrm{ml}$; cytochalasin $\mathrm{D}, 1 \mu \mathrm{g} / \mathrm{ml} ; \mathrm{NiCl}_{2}$, $2 \mathrm{mM}$ ) for $30 \mathrm{~min}$, and the inhibitors remained in the media during incubation with vesicles. All subsequent steps were carried out on ice and ice-cold Dulbecco's phosphate-buffered saline (PBS) was used for washes.
Following incubation with vesicles, cells were washed twice to remove unbound vesicles. Cell exteriors were labeled in one of two ways, as indicated in figure legends: 1) Cells were incubated with AF633-conjugated wheat germ agglutinin (WGA; $25 \mathrm{~min}$, on ice) and washed twice, or 2) Cells were incubated with 6-((biotinoyl)amino)hexanoic acid, succinimidyl ester (Biotin-X, SE; $10 \mathrm{~min}$, on ice), washed twice, and then incubated with AF633-conjugated streptavidin (15 min, on ice) and washed twice. Cells were then fixed in 2\% paraformaldehyde, mounted with ProLong AntiFade reagent, and visualized on a Nikon Eclipse TE200.

\section{Immunofluorescence}

Clathrin and caveolin immunofluorescence was performed essentially as described [14]. Following incubation with vesicles, monolayers were washed, cell exteriors were labeled with Biotin-X, SE/AF633-Streptavidin and fixed as described above. Fixed cells were washed, permeabilized $(0.1 \%$ Triton X-100 in Hanks buffer; $15 \mathrm{~min}$, $25^{\circ} \mathrm{C}$ ), blocked ( $5 \%$ goat serum and $0.1 \%$ bovine serum albumin in permeabilization buffer; $20 \mathrm{~min}, 25^{\circ} \mathrm{C}$ ), incubated with mouse anti-caveolin-1 or anti-clathrin antibodies (BD Biosciences; $2.5 \mu \mathrm{g} / \mathrm{ml}$ in permeabilization buffer; $1 \mathrm{~h}, 25^{\circ} \mathrm{C}$ ), washed, and then labeled with AF555conjugated goat anti-mouse secondary antibody $(\mu \mathrm{g} / \mathrm{ml}$ in permeabilization buffer; $30 \mathrm{~min}, 25^{\circ} \mathrm{C}$ ), and washed. Following incubation with secondary antibodies, slides were mounted and visualized as described above.

For TRAP $\alpha$ and tubulin immunofluorescence, fixed monolayers were permeabilized in PBS supplemented with 1 $\mathrm{mM}$ DTT, $1 \mathrm{mM}$ PMSF, and $0.015 \%$ digitonin (to release cytoplasmic contents) for $5 \mathrm{~min}$. Permeabilized cells were blocked with $1 \%$ BSA in PBS (30 min, on ice), incubated with rabbit anti-TRAP $\alpha$ or mouse anti- $\beta$-tubulin primary antibodies $(2 \mu \mathrm{g} / \mathrm{ml}$, in blocking buffer, $1 \mathrm{~h}$, on ice),

Table I: Primers used in these study (5' to 3 ' sequence)

\section{PA2939}

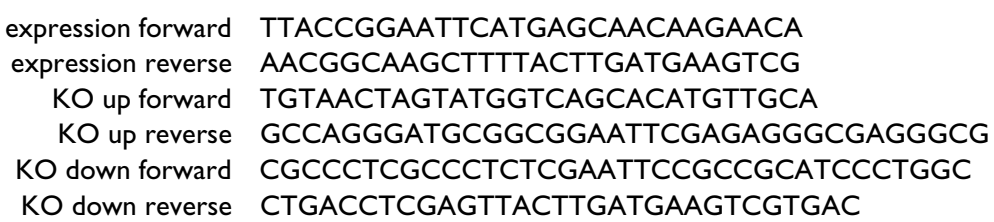

Tet $^{R}$ cassette from pACYCI 84

EcoRI Tet forward EcoRI Tet reverse Xho Tet forward Xho Tet reverse
GGTTATGAATTCGGTAGCTCAGAGAACCCTTCG GTGTTAGAATTCGATATGTTCTGCCAAGGGTT CCGGCTCGAGGGTAGCTCAGAGAACCTTCG CCGGCTCGAGGATATGTTCTGCCAAGGGTT 

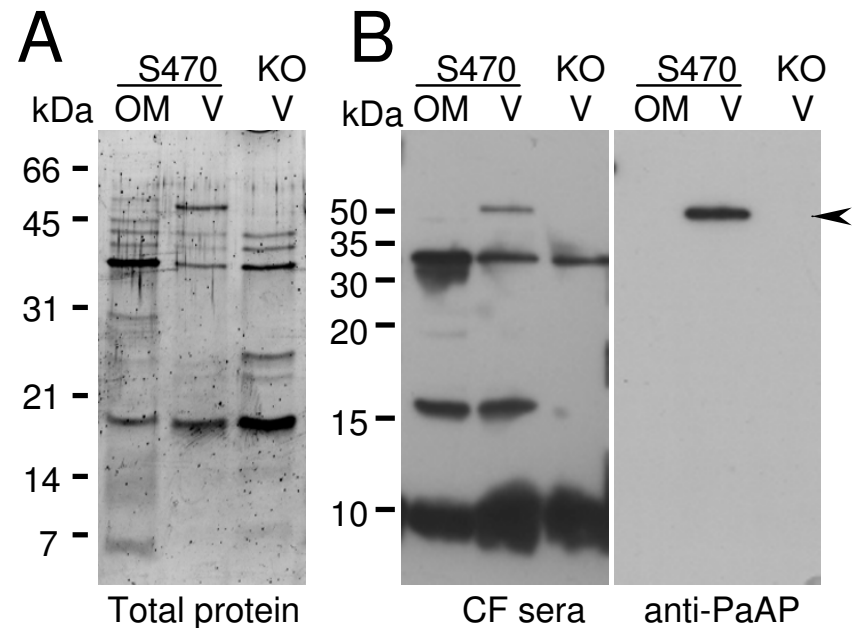

Figure 7

CF patients produce antibodies to $\mathrm{PaAP}$. Purified outer membranes (OM) from S470 and vesicles (V) from S470 and S470APKO5 (KO) $(2 \mu \mathrm{g})$ were separated by SDS-PAGE and stained with SYPRO Ruby (A) or transferred to PVDF and immunoblotted using sera from a CF patient and then reblotted with anti-PaAP $(B)$. Molecular weight standards $(\mathrm{kDa})$ and the migration of PaAP (arrow) are indicated.

washed, and incubated with AF555-conjugated goat antimouse or anti-rabbit secondary antibodies $(30 \mathrm{~min}$, on ice). Following incubation with secondary antibody, slides were mounted and visualized as described above.

\section{Leucine aminopeptidase assay}

Assays were performed using the substrate Leu-p-nitroanilide $\left(0.6 \mathrm{mM}\right.$ in $50 \mathrm{mM}$ Tris- $\left.\mathrm{HCl}, 1 \mathrm{mM} \mathrm{CaCl}_{2}, \mathrm{pH} 8.3\right)$ as described previously [44]. Samples were preincubated with $0.5 \mathrm{mM}$ dithiothreitol $\left(30 \mathrm{~min}, 25^{\circ} \mathrm{C}\right.$ ) in controls experiments (data not shown) to demonstrate specificity. The production of $\mathrm{p}$-nitroaniline ( $\mathrm{pNA}$ ) was monitored at $405 \mathrm{~nm}$.

\section{Detection of PaAP antibodies in sera from CF patients}

Outer membranes were purified as described [46,47]. Briefly, cells were harvested in stationary phase, resuspended (20\% sucrose in $30 \mathrm{mM}$ Tris, $\mathrm{pH} 8$ ), treated with DNase I and RNaseA and broken by French Press. Membranes were separated using a sucrose gradient. Purified S470 outer membrane and vesicles $(2 \mu \mathrm{g})$ were separated by SDS-PAGE and SYPRO Ruby protein stained or transferred to PVDF, immunoblotted using sera (1:10 dilution) from anonymous CF patients or anti-PaAP antibodies, and developed with SuperSignal (Pierce).

\section{Authors' contributions}

S.J.B. was responsible for designing and carrying out the experiments, M.J.K. was responsible for overseeing the research design and funding, both authors participated in data interpretation and writing of the manuscript.

\section{Additional material}

\section{Additional file 1}

Vesicles primarily colocalize with CT and transferrin in peri-nuclear regions. The data show fluorescently labeled $\$ 470$ vesicles colocalize with CT and transferrin in perinuclear regions of A549 cells.

Click here for file

[http://www.biomedcentral.com/content/supplementary/14712180-9-26-S1.pdf]

\section{Additional file 2}

PaAP contributes to the cell association of vesicles in a dose-dependent manner. The data show the amount of PaAP on vesicles correlates with the amount of vesicle association with A549 cells.

Click here for file

[http://www.biomedcentral.com/content/supplementary/1471-

2180-9-26-S2.pdf]

\section{Additional file 3}

Vesicle expression and activity of S470APKO5 complemented with plasmid-expressed PaAP. The data show the lack of PaAP activity in the APKO5 strain, the correlation between secreted aminopeptidase activity of the complemented strain with the amount of PaAP secreted, and that induced, plasmid-expressed PaAP in APKO5 is secreted to the same extent as $\$ 470$ but is not vesicle-associated.

Click here for file

[http://www.biomedcentral.com/content/supplementary/14712180-9-26-S3.pdf]

\section{Acknowledgements}

We thank J. R. Wright (Duke University) for P. aeruginosa strain S470, C.R.H. Raetz for strain MT6I6 and plasmid pJQ200SK, Erich Lanka for plasmid pMMB66EH, and M. Knowles (Adult CF Genetic Modifier Study, UNCChapel Hill, NC) for providing CF patient sera, Andy Ghio for providing HBE cells (EPA, NC), Chris Nicchitta (Duke Univeristy Medical Center) for TRAP $\alpha$ and $\beta$-tubulin antibodies, and David FitzGerald $(\mathrm{NCl}$, Bethesda, $\mathrm{MD}$ ) for monoclonal anti-PaAP antibody. We also thank J. Rudolph and $\mathrm{T}$. Hsieh for equipment use, and J.L. Plank for troubleshooting. This work was supported by a Burroughs Wellcome Investigator in Pathogenesis of Infectious Disease Award (to M.J.K.), an American Lung Association research grant, the Cystic Fibrosis Foundation, the Thomas H. Davis Research Award of the ALA of NC, and the N.I.H.

\section{References}

I. Yoon SS, Hennigan RF, Hilliard GM, Ochsner UA, Parvatiyar K, Kamani MC, Allen HL, DeKievit TR, Gardner PR, Schwab U, Rowe إل, Iglewski BH, McDermott TR, Mason RP, Wozniak DJ, Hancock RE, Parsek MR, Noah TL, Boucher RC, Hassett DJ: Pseudomonas aeruginosa anaerobic respiration in biofilms: relationships to cystic fibrosis pathogenesis. Developmental cell 2002, 3(4):593-603.

2. Worlitzsch D, Tarran R, Ulrich M, Schwab U, Cekici A, Meyer KC, Birrer P, Bellon G, Berger J, Weiss T, Botzenhart K, Yankaskas JR, Randell S, Boucher RC, Doring G: Effects of reduced mucus oxygen concentration in airway Pseudomonas infections of cystic fibrosis patients. The Journal of clinical investigation 2002, I09(3):317-325. 
3. Wilderman PJ, Vasil AI, Johnson Z, Wilson MJ, Cunliffe HE, Lamont IL, Vasil ML: Characterization of an endoprotease (PrpL) encoded by a PrdS-regulated gene in Pseudomonas aeruginosa. Infection and immunity 200I, 69(9):5385-5394.

4. Van Delden C, Iglewski BH: Cell-to-cell signaling and Pseudomonas aeruginosa infections. Emerging infectious diseases 1998 4(4):55I-560.

5. Malloy JL, Veldhuizen RA, Thibodeaux BA, O'Callaghan RJ, Wright JR: Pseudomonas aeruginosa protease IV degrades surfactant proteins and inhibits surfactant host defense and biophysical functions. American journal of physiology 2005, 288(2): L409-418.

6. Beveridge TJ: Structures of gram-negative cell walls and their derived membrane vesicles. Journal of bacteriology 1999, I 8 I ( 16):4725-4733

7. McBroom A, Kuehn MJ: Chapter 2.2.4 Outer membrane vesicles. In EcoSal - Escherichia coli and Salmonella: Cellular and Molecular Biology [Online] Edited by: Curtiss R III. Washington, D.C.: ASM Press; 2005.

8. Bauman SJ, Kuehn MJ: Purification of outer membrane vesicles from Pseudomonas aeruginosa and their activation of an IL8 response. Microbes Infect 2006, 8(9-10):2400-2408.

9. Kuehn MJ, Kesty NC: Bacterial outer membrane vesicles and the host-pathogen interaction. Genes Dev 2005, 19(22):2645-2655.

10. Keenan J, Day T, Neal S, Cook B, Perez-Perez G, Allardyce R, Bagshaw $\mathrm{P}$ : A role for the bacterial outer membrane in the pathogenesis of Helicobacter pylori infection. FEMS microbiology letters 2000, I 82(2):259-264.

II. Horstman AL, Kuehn MJ: Enterotoxigenic Escherichia coli secretes active heat-labile enterotoxin via outer membrane vesicles. The Journal of biological chemistry 2000 275(I 7): 12489-12496.

12. Wai SN, Lindmark B, Soderblom T, Takade A, Westermark M, Oscarsson J, Jass J, Richter-Dahlfors A, Mizunoe Y, Uhlin BE: Vesiclemediated export and assembly of pore-forming oligomers of the enterobacterial ClyA cytotoxin. Cell 2003, I I 5(I):25-35

13. Demuth DR, James D, Kowashi Y, Kato S: Interaction of Actinobacillus actinomycetemcomitans outer membrane vesicles with HL60 cells does not require leukotoxin. Cell Microbio 2003, 5(2): || II-|2|.

14. Kesty NC, Mason KM, Reedy M, Miller SE, Kuehn MJ: Enterotoxigenic Escherichia coli vesicles target toxin delivery into mammalian cells. EMBO J 2004, 23(23):4538-4549.

15. Balsalobre C, Silvan JM, Berglund S, Mizunoe Y, Uhlin BE, Wai SN: Release of the type I secreted alpha-haemolysin via outer membrane vesicles from Escherichia coli. Molecular microbiology 2006, 59(I):99-112.

16. Nowotny A, Behling UH, Hammond B, Lai CH, Listgarten M, Pham $\mathrm{PH}$, Sanavi F: Release of toxic microvesicles by Actinobacillus actinomycetemcomitans. Infect Immun 1982, 37(I): I5I-I54.

17. Kadurugamuwa JL, Beveridge TJ: Delivery of the non-membranepermeative antibiotic gentamicin into mammalian cells by using Shigella flexneri membrane vesicles. Antimicrob Agents Chemother 1998, 42(6): | 476-I 483 .

18. Shoberg RJ, Thomas DD: Specific adherence of Borrelia burgdorferi extracellular vesicles to human endothelial cells in culture. Infect Immun 1993, 6 I(9):3892-3900.

19. Kato S, Kowashi Y, Demuth DR: Outer membrane-like vesicles secreted by Actinobacillus actinomycetemcomitans are enriched in leukotoxin. Microbial pathogenesis 2002, 32(1): I-I3.

20. Kesty NC, Kuehn MJ: Incorporation of Heterologous Outer Membrane and Periplasmic Proteins into Escherichia coli Outer Membrane Vesicles. J Biol Chem 2004, 279(3):2069-2076.

21. Heczko U, Smith VC, Mark Meloche R, Buchan AM, Finlay BB: Characteristics of Helicobacter pylori attachment to human primary antral epithelial cells. Microbes Infect 2000, 2(14):1669-1676

22. Kadurugamuwa JL, Beveridge TJ: Virulence factors are released from Pseudomonas aeruginosa in association with membrane vesicles during normal growth and exposure to gentamicin: a novel mechanism of enzyme secretion. Journal of bacteriology 1995, 177(14):3998-4008.

23. Kadurugamuwa JL, Beveridge TJ: Natural release of virulence factors in membrane vesicles by Pseudomonas aeruginosa and the effect of aminoglycoside antibiotics on their release. Antimicrob Chemother 1997, 40(5):615-621.
24. Mashburn LM, Whiteley M: Membrane vesicles traffic signals and facilitate group activities in a prokaryote. Nature 2005, 437(7057):422-425.

25. Alvarez-Ortega C, Harwood CS: Responses of Pseudomonas aeruginosa to low oxygen indicate that growth in the cystic fibrosis lung is by aerobic respiration. Molecular microbiology 2007, 65(I): 153-165.

26. Chugani S, Greenberg EP: The influence of human respiratory epithelia on Pseudomonas aeruginosa gene expression. Microb Pathog 2007, 42(I):29-35.

27. Corbett CR, Burtnick MN, Kooi C, Woods DE, Sokol PA: An extracellular zinc metalloprotease gene of Burkholderia cepacia. Microbiology 2003, I49(Pt 8):2263-227I.

28. Rodal SK, Skretting G, Garred O, Vilhardt F, van Deurs B, Sandvig K: Extraction of cholesterol with methyl-beta-cyclodextrin perturbs formation of clathrin-coated endocytic vesicles. Molecular biology of the cell 1999, 10(4):961-974.

29. Heuser JE, Anderson RG: Hypertonic media inhibit receptormediated endocytosis by blocking clathrin-coated pit formation. The Journal of cell biology 1989, 108(2):389-400.

30. Yang CP, Galbiati F, Volonte D, Horwitz SB, Lisanti MP: Upregulation of caveolin-I and caveolae organelles in Taxol-resistant A549 cells. FEBS letters 1998, 439(3):368-372.

3I. Campbell L, Hollins AJ, Al-Eid A, Newman GR, von Ruhland C, Gumbleton M: Caveolin-I expression and caveolae biogenesis during cell transdifferentiation in lung alveolar epithelial primary cultures. Biochemical and biophysical research communications 1999, 262(3):744-75।.

32. Lerner RS, Seiser RM, Zheng T, Lager PJ, Reedy MC, Keene JD, Nicchitta CV: Partitioning and translation of mRNAs encoding soluble proteins on membrane-bound ribosomes. RNA 2003 , 9(9): I I 23- I I37.

33. Stephens SB, Dodd RD, Brewer JW, Lager PJ, Keene JD, Nicchitta CV: Stable ribosome binding to the endoplasmic reticulum enables compartment-specific regulation of mRNA translation. Molecular biology of the cell 2005, I 6( I 2):58| 9-583।.

34. Tsuda K, Amano A, Umebayashi K, Inaba H, Nakagawa I, Nakanishi Y, Yoshimori T: Molecular dissection of internalization of Porphyromonas gingivalis by cells using fluorescent beads coated with bacterial membrane vesicle. Cell structure and function 2005, 30(2):8I-9I.

35. Grassme H, Jendrossek V, Riehle A, von Kurthy G, Berger J, Schwarz $\mathrm{H}$, Weller M, Kolesnick R, Gulbins E: Host defense against Pseudomonas aeruginosa requires ceramide-rich membrane rafts. Nature medicine 2003, 9(3):322-330.

36. Kadurugamuwa JL, Beveridge TJ: Delivery of the non-membranepermeative antibiotic gentamicin into mammalian cells by using Shigella flexneri membrane vesicles. Antimicrob Agents Chemother 1998, 42(6): | 1476-1483.

37. Wagner VE, Li LL, Isabella VM, Iglewski BH: Analysis of the hierarchy of quorum-sensing regulation in Pseudomonas aeruginosa. Analytical and bioanalytical chemistry 2007, 387(2):469-479.

38. Schuster M, Lostroh CP, Ogi T, Greenberg EP: Identification, timing, and signal specificity of Pseudomonas aeruginosa quorum-controlled genes: a transcriptome analysis. Journal of bacteriology 2003, I85(7):2066-2079.

39. Nouwens AS, Beatson SA, Whitchurch CB, Walsh BJ, Schweizer HP, Mattick JS, Cordwell SJ: Proteome analysis of extracellular proteins regulated by the las and rhl quorum sensing systems in Pseudomonas aeruginosa PAOI. Microbiology (Reading, England) 2003, I49(Pt 5): |3||-|322.

40. Schuster M, Hawkins AC, Harwood CS, Greenberg EP: The Pseudomonas aeruginosa RpoS regulon and its relationship to quorum sensing. Molecular microbiology 2004, 5 I (4):973-985.

4I. Engel LS, Hobden JA, Moreau JM, Callegan MC, Hill JM, O'Callaghan $\mathrm{RJ}$ : Pseudomonas deficient in protease IV has significantly reduced corneal virulence. Investigative ophthalmology \& visual science 1997, 38(8): 1535-1542.

42. Preston MJ, Seed PC, Toder DS, Iglewski BH, Ohman DE, Gustin JK, Goldberg JB, Pier GB: Contribution of proteases and LasR to the virulence of Pseudomonas aeruginosa during corneal infections. Infect Immun 1997, 65(8):3086-3090.

43. Engel LS, Hill JM, Moreau JM, Green LC, Hobden JA, O'Callaghan RJ: Pseudomonas aeruginosa protease IV produces corneal damage and contributes to bacterial virulence. Investigative ophthalmology \& visual science 1998, 39(3):662-665. 
44. Cahan R, Axelrad I, Safrin M, Ohman DE, Kessler E: A secreted aminopeptidase of Pseudomonas aeruginosa. Identification, primary structure, and relationship to other aminopeptidases. J Biol Chem 200 I, 276(47):43645-43652.

45. Goldberg JB, Ohman DE: Cloning and expression in Pseudomonas aeruginosa of a gene involved in the production of alginate. Journal of bacteriology 1984, I 58(3): I | |5-II2I.

46. Hancock RE, Nikaido $H$ : Outer membranes of gram-negative bacteria. XIX. Isolation from Pseudomonas aeruginosa $P A O I$ and use in reconstitution and definition of the permeability barrier. J Bacteriol 1978, 136(1):38I-390.

47. Hancock Laboratory Methods [http://www.cmdr.ubc.ca/bobh/ methodsall.html]

Publish with Bio Med Central and every scientist can read your work free of charge

"BioMed Central will be the most significant development for disseminating the results of biomedical research in our lifetime. "

Sir Paul Nurse, Cancer Research UK

Your research papers will be:

- available free of charge to the entire biomedical community

- peer reviewed and published immediately upon acceptance

- cited in PubMed and archived on PubMed Central

- yours - you keep the copyright
BioMedcentral 